\title{
Design of Harmonic AFM Probe Subjected to van der Waals Force in the Modified Couple Stress Theory
}

\author{
Shueei-Muh Lin, Ching-Yao Chang, Chihng-Tsung Liauh, and Wen-Rong Wang \\ Mechanical Engineering Department, Kun Shan University and Green Energy Technology Research Centre (GETRC), \\ Tainan 710-03, Taiwan, China \\ Correspondence should be addressed to Shueei-Muh Lin; smlin45@gmail.com
}

Received 6 December 2020; Revised 26 February 2021; Accepted 5 March 2021; Published 26 March 2021

Academic Editor: Kai Zhang

Copyright (c) 2021 Shueei-Muh Lin et al. This is an open access article distributed under the Creative Commons Attribution License, which permits unrestricted use, distribution, and reproduction in any medium, provided the original work is properly cited.

The conventional design of harmonic AFM probe geometry is made in neglect of the effects of the size-dependency factor and the tip-sample interacting force. Obviously, the effect of these two factors on the natural frequencies of a probe is significant. In this study, the effects of the two factors on the integer-multiples relation among frequencies are investigated. In this study, the effects of the two factors on the integer-multiples relation among frequencies are investigated. It is discovered that, in general, the integermultiples relations of the probe's frequencies in the classical model does not be kept as the same as that in the system with the effect of the size-dependency factor under the same material and geometry properties of probe. In addition, when the probe is used to measure the sample, the deviation of the relations will happen. The smaller the tip-sample distance is, the larger the deviation of integer-multiples frequencies is. The analytical method is presented here such that during scanning a sample at some tip-sample distance, the material and geometry properties of the probe can be tuned to the integer-multiples relation of resonant frequencies. Moreover, five similarity conditions among the systems with and without the effects of size-dependency and the tip-sample interacting force are discovered. According to these conditions, the integer-multiples relation is kept in different systems.

\section{Introduction}

For high-speed AFM imaging, atomic force microscopy is often employed to measure the surface topography and material property of specimens, simultaneously. The dynamic response of the fundamental mode is conventionally used to measure the topography of the sample. The response of higher flexural modes of a cantilever is taken to measure some material properties [1-6]. It is found that if the probe's higher-order resonance frequencies match the integer multiples of its fundamental frequency, the probe's responses at such harmonic frequencies will be enhanced. The harmonic amplitude is sensitive to local mechanical properties of the specimen [5-7]. In general, the design methods of a harmonic probe include (1) the attached mass method [8], (2) the removed mass method $[6,9,10],(3)$ the cutting-boundary method $[5,11,12]$, and (4) the varying cross section method [13]. The literatures are introduced as follows.

Li et al. [8] proposed attaching micro/nanoparticles with specific mass and position on the cantilever. Unfortunately, precise positioning of the attached mass could be technically not so easy to realize. The literatures $[6,9,10]$ investigated the selective removal of the lever materials is more convenient than the previous one, which can be accomplished by changing the cantilever shape and cutting hole structures in the probe. The literatures $[5,11,12]$ studied the cutting the boundary of probe cross section to tailor the frequency characteristics. In the above literatures, the finite element method is used to determine the frequencies of the probe due to no general rule about the geometry. Moreover, the method is just for some special case. For example, Cai et al. [11] changed the geometry of the commercial probe to tune the integer-multiples relation. In the literatures $[5,6,8-12]$, the classical theory of elasticity is used to investigate the harmonic AFM probe. Moreover, the effect of structure's size dependency is not considered. Lin and Chang [13] designed the harmonic AFM probe with varying cross section in polynomial series based on the modified couple stress theory, neglecting the effect of the tip-sample interacting force. However, it is hard to manufacture the varying 
cross section of the probe precisely due to the complicated geometry. In addition, the integer-multiples relations without the effect of the tip-sample interacting force will not be kept during scanning the sample.

Several literatures [14-17] discovered that when the structures are of the order of microns or submicrons, the size-dependent effect on the behavior of structure is significant. Some literatures investigated mechanical properties of nanostructures in nonclassical theories. These theories are (1) the couple stress theory $[18,19],(2)$ the strain gradient theory [20], (3) the nonlocal theory [21], and (4) the surface elasticity model [22]. Lin et al. [18, 19] investigated the dynamic behaviors of a conventional AFM uniform probe subjected to the van der Waals force and the multimode excitation based on the modified couple stress theory. Further, Lin et al. [20] made assessment of the classical, modified couple stress, and strain gradient theories about size dependency effect on the resonant frequency of a conventional AFM uniform probe subjected to van der Waals force. The literatures [18-20] investigated the dynamic behavior of the conventional probe with the size dependency effect only.

So far, no literature is devoted to design the harmonic probe with the integer-multiples relation of frequencies considering the effects of the size dependency and the tipsample interacting force during an AFM probe measuring a sample's topography and properties. In this study, the design of linearly varying cross section of the probe with the integer-multiples relation of frequencies and these effects is proposed. The cross section of the probe is easily and precisely manufactured. Moreover, the similarity conditions among the systems with and without the effects of sizedependency and the tip-sample interacting force are investigated.

\section{Mathematical Model in the Modified Couple Stress Theory}

2.1. Governing Equation and Boundary Conditions in Hamilton's Principle. In this study, the conventional AFM slender probe has a large aspect ratio and its deflection during measuring the topography of the sample is very small; the Bernoulli-Euler beam theory is usually used to simulate the system. The dynamic response of a Bernoulli-Euler beam with tip mass subjected to the van der Waals interacting force $F_{v}$ is investigated, as shown in Figure 1.

Application of Hamilton's principle [13, 23] yields the coupled governing differential equations and the associated general elastic boundary conditions. The governing equation of an AFM cantilever probe as shown in Figure 1 is

$$
\frac{\partial^{2}}{\partial x^{2}}\left[\left(E I+\mu A \ell_{c}^{2}\right) \frac{\partial^{2} W}{\partial x^{2}}\right]+\rho A \frac{\partial^{2} W}{\partial t^{2}}=0, \quad 0<x<L .
$$

The clamped boundary conditions are expressed as follows.

$$
\text { At } x=0 \text { : }
$$

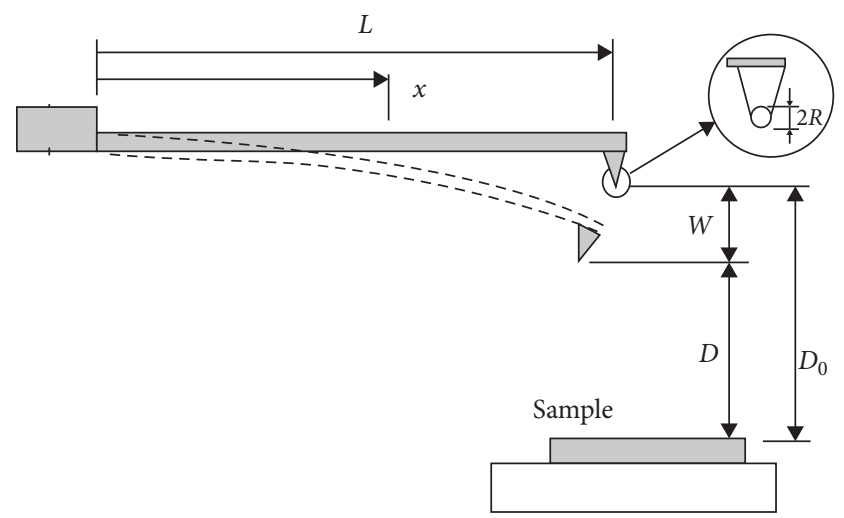

Figure 1: Geometry and coordinate system of a micro probe.

$$
\begin{aligned}
W & =0, \\
\frac{\partial W}{\partial x} & =0 .
\end{aligned}
$$

At $x=L$ :

$$
\begin{aligned}
\frac{\partial^{2} W}{\partial x^{2}} & =0, \\
\frac{\partial}{\partial x}\left[\left(E I+\mu A \ell_{c}^{2}\right) \frac{\partial^{2} W}{\partial x^{2}}\right]-m_{\mathrm{tip}} \frac{\partial^{2} W(L, t)}{\partial t^{2}} & =F_{v},
\end{aligned}
$$

where $F_{v}$ is the van der Waals tip-sample interacting force [5] which is expressed as

$$
F_{v}=-\frac{A_{H} R}{6 D^{2}}
$$

in which the tip-surface distance $D=D_{0}-W(L, t), A_{H}$ is the Hamaker constant, $D_{0}$ is the distance between the tip of the undeformed beam and the surface of sample, and $R$ is the tip radius, as shown in Figure 1.

\subsection{Dimensionless Governing Equation and Boundary} Conditions. In terms of the dimensionless parameters in the nomenclature, the dimensionless governing equation and boundary conditions are

$$
\frac{\partial^{2}}{\partial \xi^{2}}\left\{[b(\xi)+\delta m(\xi)] \frac{\partial^{2} w}{\partial \xi^{2}}\right\}+m(\xi) \frac{\partial^{2} w}{\partial \tau^{2}}=0, \quad \xi \in(0,1) .
$$

The clamped boundary conditions are expressed as follows.

At $\xi=0$ :

$$
\begin{gathered}
w=0, \\
\frac{\partial w}{\partial \xi}=0 .
\end{gathered}
$$

At $\xi=1$ : 


$$
\begin{array}{r}
\frac{\partial^{2} w}{\partial \xi^{2}}=0 \\
\frac{\partial}{\partial \xi}\left[(b(\xi)+\delta m(\xi)) \frac{\partial^{2} w}{\partial \xi^{2}}\right]-\mu_{\mathrm{tip}} \frac{\partial^{2} w}{\partial \tau^{2}}=f_{v}
\end{array}
$$

where $f_{v}=-\left(c_{v} / 6 d^{2}\right)$ and the tip-surface distance is

$$
d=1-w(1, \tau)
$$

Because boundary condition (16) is nonlinear, it is hard to derive the solution of the nonlinear system. In this study, the analytical method is proposed.

\section{Solution Method}

3.1. Separation of Variable. The dynamic solution of harmonic motion is

$$
w(\xi, \tau)=\bar{w}(\xi) \cos \omega \tau .
$$

Substituting equation (11) into governing equation (5) and boundary conditions (6)-(8), the transformed governing equation is obtained.

$$
\frac{\mathrm{d}^{2}}{\mathrm{~d} \xi^{2}}\left[[b(\xi)+\delta m(\xi)] \frac{\mathrm{d}^{2} \bar{w}}{\mathrm{~d} \xi^{2}}\right]-m(\xi) \omega^{2} \bar{w}=0, \quad \xi \in(0,1)
$$

and the associated boundary conditions are expressed as follows.

At $\xi=0$ :

$$
\begin{aligned}
\bar{w} & =0, \\
\frac{\mathrm{d} \bar{w}}{\mathrm{~d} \xi} & =0 .
\end{aligned}
$$

At $\xi=1$ :

$$
\frac{\mathrm{d}^{2} \bar{w}}{\mathrm{~d} \xi^{2}}=0
$$

Further, substituting equation (11) into boundary condition (9) and multiplying it by $\cos (\omega \tau)$ and integrating it from 0 to the period $T,(2 \pi / \omega)$, equation (9) becomes

$$
\frac{\mathrm{d}}{\mathrm{d} \xi}\left[(b(\xi)+\delta m(\xi)) \frac{\mathrm{d}^{2} \bar{w}}{\mathrm{~d} \xi^{2}}\right]+\mu_{\text {tip }} \omega^{2} \bar{w}=\bar{f}_{v}^{*},
$$

where $\bar{f}_{v}^{*}=-\left(c_{v} \bar{w}(1) / 3\left(1-\bar{w}^{2}(1)\right)^{(3 / 2)}\right)$.

So far, the linear characteristic system composed of equations (12)-(16) is obtained. Further, it can be solved as follows.

3.2. Frequency Equation. The solution of the characteristic governing equation (12) can be written as

$$
\bar{w}=C_{1} V_{1}(\xi)+C_{2} V_{2}(\xi)+C_{3} V_{3}(\xi)+C_{4} V_{4}(\xi),
$$

where $V_{i}(\xi), i=1,2,3,4$ are the four linearly independent fundamental solutions of equation (14), which are assumed to satisfy the following normalized condition:

$$
\left[\begin{array}{cccc}
V_{1}(0) & V_{2}(0) & V_{3}(0) & V_{4}(0) \\
V_{1}^{\prime}(0) & V_{2}^{\prime}(0) & V_{3}^{\prime}(0) & V_{4}^{\prime}(0) \\
V_{1}^{\prime \prime}(0) & V_{2}^{\prime \prime}(0) & V_{3}^{\prime \prime}(0) & V_{4}^{\prime \prime}(0) \\
V_{1}^{\prime \prime \prime}(0) & V_{2}^{\prime \prime \prime}(0) & V_{3}^{\prime \prime \prime}(0) & V_{4}^{\prime \prime \prime}(0)
\end{array}\right]=\left[\begin{array}{llll}
1 & 0 & 0 & 0 \\
0 & 1 & 0 & 0 \\
0 & 0 & 1 & 0 \\
0 & 0 & 0 & 1
\end{array}\right] .
$$

The coefficient $C_{i}, i=1,2,3,4$, is dependent on characteristic boundary conditions (13)-(16). Substituting equation (17) into boundary conditions (13)-(16), the amplitude is

$$
\bar{w}(\xi)=C_{3} V_{3}(\xi)+C_{4} V_{4}(\xi)
$$

where

$$
\begin{aligned}
C_{4}= & \frac{-V_{3}^{\prime \prime}(1)}{V_{4}^{\prime \prime}(1)} C_{3}, \\
C_{3}= & \frac{f_{v}^{*}}{\beta}, \\
\beta= & {\left[(b(1)+\delta m(1)) V_{3}^{\prime \prime \prime}(1)+\left(b^{\prime}(1)+\delta m^{\prime}(1)\right) V_{3}^{\prime \prime}(1)+\mu_{\text {tip }} \omega^{2} V_{3}(1)\right] } \\
& -\frac{V_{3}^{\prime \prime}(1)}{V_{4}^{\prime \prime}(1)}\left[(b(1)+\delta m(1)) V_{4}^{\prime \prime \prime}(1)+\left(b^{\prime}(1)+\delta m^{\prime}(1)\right) V_{4}^{\prime \prime}(1)+\mu_{\text {tip }} \omega^{2} V_{4}(1)\right] .
\end{aligned}
$$

The associated frequency equation is

$$
\left|\bar{w}_{\text {calculated }}(1)-\bar{w}_{\text {given }}(1)\right| \longrightarrow 0 \text {. }
$$

It is well known that the resonant frequency depends significantly on the amplitude of probe vibration $\bar{w}_{\text {given }}(1)$.
One can determine the resonant frequency via frequency equation (21).

3.3. Semianalytical Fundamental Solutions. The length of the beam is divided into several continuous subdomains as 
shown in Figure 2. The displacement in the $(j+1)$ th subdomain can be expressed in terms of the global fundamental solutions $V_{i}$ and local ones $\bar{V}_{j+1, i}$ :

$$
\begin{aligned}
\bar{w}^{(k)}(\xi) & =C_{1} V_{1}^{(k)}(\xi)+C_{2} V_{2}^{(k)}(\xi)+C_{3} V_{3}^{(k)}(\xi)+C_{4} V_{4}^{(k)}(\xi) \\
& =C_{j+1,1} \bar{V}_{j+1,1}^{(k)}(\xi)+C_{j+1,2} \bar{V}_{j+1,2}^{(k)}(\xi)+C_{j+1,3} \bar{V}_{j+1,3}^{(k)}(\xi)+C_{j+1,4} \bar{V}_{j+1,4}^{(k)}(\xi), \quad \xi \in\left(\xi_{j}, \xi_{j+1}\right), k=0,1,2,3
\end{aligned}
$$

The local fundamental solutions in the $(j+1)$ th subdomain satisfy the following normalized condition:

$$
\left[\begin{array}{llll}
\bar{V}_{j+1,1} & \bar{V}_{j+1,2} & \bar{V}_{j+1,3} & \bar{V}_{j+1,4} \\
\bar{V}_{j+1,1}^{(1)} & \bar{V}_{j+1,2}^{(1)} & \bar{V}_{j+1,3}^{(1)} & \bar{V}_{j+1,4}^{(1)} \\
\bar{V}_{j+1,1}^{(2)} & \bar{V}_{j+1,2}^{(2)} & \bar{V}_{j+1,3}^{(3)} & \bar{V}_{j+1,4}^{(4)} \\
\bar{V}_{j+1,1}^{(3)} & \bar{V}_{j+1,2}^{(3)} & \bar{V}_{j+1,3}^{(3)} & \bar{V}_{j+1,4}^{(3)}
\end{array}\right]_{\xi=\xi_{j}}=\left[\begin{array}{llll}
1 & 0 & 0 & 0 \\
0 & 1 & 0 & 0 \\
0 & 0 & 1 & 0 \\
0 & 0 & 0 & 1
\end{array}\right] .
$$

The four continuity conditions at the interface between two neighboring subdomains are

$$
\begin{aligned}
\bar{w}\left(\xi_{j}^{-}\right) & =\bar{w}\left(\xi_{j}^{+}\right), \\
\bar{w}^{(1)}\left(\xi_{j}^{-}\right) & =\bar{w}^{(1)}\left(\xi_{j}^{+}\right), \\
\bar{w}^{(2)}\left(\xi_{j}^{-}\right) & =\bar{w}^{(2)}\left(\xi_{j}^{+}\right), \\
\bar{w}^{(3)}\left(\xi_{j}^{-}\right) & =\bar{w}^{(3)}\left(\xi_{j}^{+}\right) .
\end{aligned}
$$

Substituting equation (22) into continuity condition (24), the coefficients of the $(j+1)$ th subdomain can be expressed in the fundamental solutions of the $j$-th subdomain:

$$
\begin{aligned}
& C_{j+1,1}=\bar{w}\left(\xi_{j}^{-}\right), \\
& C_{j+1,2}=\bar{w}^{(1)}\left(\xi_{j}^{-}\right), \\
& C_{j+1,3}=\bar{w}^{(2)}\left(\xi_{j}^{-}\right), \\
& C_{j+1,4}=\bar{w}^{(3)}\left(\xi_{j}^{-}\right) .
\end{aligned}
$$

Finally, the global semianalytical fundamental solutions are derived in terms of the local ones:

$$
\begin{aligned}
V_{i}(\xi) & =V_{i}^{(0)}\left(\xi_{j}\right) \bar{V}_{j+1,1}(\xi)+V_{i}^{(1)}\left(\xi_{j}\right) \bar{V}_{j+1,2}(\xi)+V_{i}^{(2)}\left(\xi_{j}\right) \bar{V}_{j+1,3}(\xi)+V_{i}^{(3)}\left(\xi_{j}\right) \bar{V}_{j+1,4}(\xi), \\
\xi & \in\left(\xi_{j}, \xi_{j+1}\right) \text { in the } j+1 \text { th section; } \quad i=1,2,3,4 ; k=1,2,3 .
\end{aligned}
$$

\section{Relationships between Resonance Frequencies and Size Parameters}

4.1. Probe with Constant Thickness in Modified Couple Stress Theory. Considering the constant thickness, $H(x)=H_{0}$, the dimensionless mass $m(\xi)$ is the same as the dimensionless bending rigidity $b(\xi)$. The associated governing equation (12) becomes

$$
\frac{\mathrm{d}^{2}}{\mathrm{~d} \xi^{2}}\left[b(\xi) \frac{\mathrm{d}^{2} \bar{w}}{\mathrm{~d} \xi^{2}}\right]-m(\xi) \bar{\omega}_{\text {stress }}^{2} \bar{w}=0, \quad \xi \in(0,1),
$$

where $\bar{\omega}_{\text {stress }}^{2}=\left(\omega_{\text {stress }}^{2} /(1+\delta)\right)$. The associated boundary conditions (13)-(16) are transformed as follows.

At $(\xi)=0$ :

$$
\begin{gathered}
\bar{w}=0, \\
\frac{\mathrm{d} \bar{w}}{\mathrm{~d} \xi}=0 .
\end{gathered}
$$

$$
\text { At }(\xi)=1 \text { : }
$$

$$
\begin{aligned}
\frac{\mathrm{d}^{2} \bar{w}}{\mathrm{~d} \xi^{2}} & =0 \\
\frac{\mathrm{d}}{\mathrm{d} \xi}\left[b(\xi) \frac{\mathrm{d}^{2} \bar{w}}{\mathrm{~d} \xi^{2}}\right]+\mu_{\text {tip }} \bar{\omega}_{\text {stress }}^{2} \bar{w} & =-\bar{c}_{v, \text { stress }} \frac{\bar{w}(1)}{3\left[1-\bar{w}^{2}(1)\right]^{(3 / 2)}} \\
& \equiv \bar{f}_{v, \text { stress }}^{*}
\end{aligned}
$$

where $\bar{c}_{v \text {,stress }} \equiv\left(c_{v} /(1+\delta)\right)$. The corresponding displacement is

$$
\bar{w}(\xi)=C_{3} V_{3}(\xi)+C_{4} V_{4}(\xi)
$$

where 


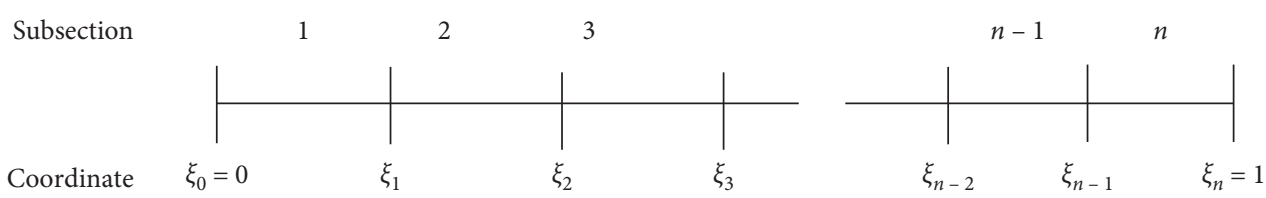

FIGURE 2: Definition of the subsections of the beam coordinate.

$$
\begin{aligned}
& C_{3}=\frac{\bar{f}_{v, \text { stress }}^{*}}{\beta}, \\
& C_{4}=\frac{-V_{3}^{\prime \prime}(1)}{V_{4}^{\prime \prime}(1)} C_{3},
\end{aligned}
$$$$
\beta=\left[b(1) V_{3}^{\prime \prime}(1)+b^{\prime}(1) V_{3}^{\prime \prime}(1)+\mu_{\text {tip }} \bar{\omega}_{\text {stress }}^{2} V_{3}(1)\right]-\frac{V_{3}^{\prime \prime}(1)}{V_{4}^{\prime \prime}(1)}\left[b(1) V_{4}^{\prime \prime}(1)+b^{\prime}(1) V_{4}^{\prime \prime}(1)+\mu_{\text {tip }} \bar{\omega}_{\text {stress }}^{2} V_{4}(1)\right]
$$$$
D_{0, \text { stress }}=D_{0, \text { classical }} \text {, }
$$$$
\omega_{\text {stress }}=\omega_{\text {classical }} \sqrt{1+\delta} .
$$

Equation (38) is the geometry similarity of the probe. Equation (39) is the tip similarity. Equations (40) and (41) are the similarity of measurement conditions. Equation (42) is the similarity of frequency.

In the absence of the effect of the tip-sample interacting force, only two similar relations (38) and (42) is required [15]. When the dimensionless bending rigidity $b(\xi)$ is the same as the dimensionless mass $m(\xi)$, one can predict the resonant frequencies in the modified couple stress theory by a similar equation (43) and the frequencies in the classical theory.

Considering the effect of the tip-sample interacting force, similar relations (38)-(41) must be satisfied. One can predict the resonant frequencies in the modified stress theory based on the frequencies in the classical theory via similarity relation (42). So far, no literature is devoted to the similarity mechanism. In reality, the effect of nano size depends on the dimension and geometry. The size-dependency parameter $\delta$ changes with different conditions and is not accurately obtained so far. In general, the integermultiples relation among frequencies depends on the sizedependency parameter $\delta$. However, if conditions (38)-(42) are satisfied, the integer-multiples relation is preserved in spite of the size-dependency parameter $\delta$.

4.3. Similarity Formula. It is found that if the dimensionless bending rigidity $b(\xi)$ is the same as the dimensionless mass $m(\xi)$, the characteristic system in the modified couple stress theory is composed of equations (33)-(37). Based on the characteristic systems in the two different theories, it is discovered that if the following relations are satisfied, the two characteristic systems are same.

$$
\begin{aligned}
b(\xi) & =m(\xi), \\
\bar{c}_{v, \text { stress }} & =\frac{c_{v, \text { stress }}}{(1+\delta)}=c_{v, \text { classical }} \\
\text { or } c_{v, \text { stress }} & =(1+\delta) c_{v, \text { classical }}, \\
w_{\text {stress }}(1) & =w_{\text {classical }},
\end{aligned}
$$

\section{Numerical Results}

Consider the stepwise tapered rectangular cross-sectional probe as shown in Figure 3. The constant thickness is $H(x)=H_{0}$. The width is

$$
B(x)=B_{0} \begin{cases}\left(1+\lambda_{1} \xi\right), & 0 \leq \xi<\xi_{1}, \\ \left(1+\lambda_{1} \xi_{1}\right)+\lambda_{2}\left(\xi-\xi_{1}\right), & \xi_{1} \leq \xi<\xi_{2}, \\ \left(1+\lambda_{1} \xi_{1}\right)+\lambda_{2}\left(\xi_{2}-\xi_{1}\right)+\lambda_{3}\left(\xi-\xi_{2}\right), & \xi_{2} \leq \xi<1 .\end{cases}
$$

The dimensionless mass and bending rigidity are 
TABLE 1: Three types of probe satisfying the integer-multiples relation among frequencies without the effects of the tip-sample force and the size dependency $\left(\mu=4.0381 \times 10^{-3}, \xi_{1}=0.4\right.$, and $\left.\xi_{2}=0.7\right)$.

\begin{tabular}{cccccccc}
\hline$\lambda_{1}$ & $\lambda_{2}$ & $\lambda_{3}$ & $\omega_{1}$ & $\omega_{2}$ & $\omega_{3}$ & $\left(\omega_{2} / \omega_{1}\right)$ & $\left(\omega_{3} / \omega_{1}\right)$ \\
\hline 1.00000 & 0.68125 & -1.72978 & 3.5370 & 21.2219 & 60.1282 & 6.000 & 17.000 \\
1.00000 & 1.72422 & -1.01995 & 3.0013 & 21.0090 & 60.0256 & 7.000 & 20.000 \\
1.00000 & 2.63750 & -0.13550 & 2.6171 & 20.9367 & 60.1932 & 8.000 & 23.000 \\
\hline
\end{tabular}

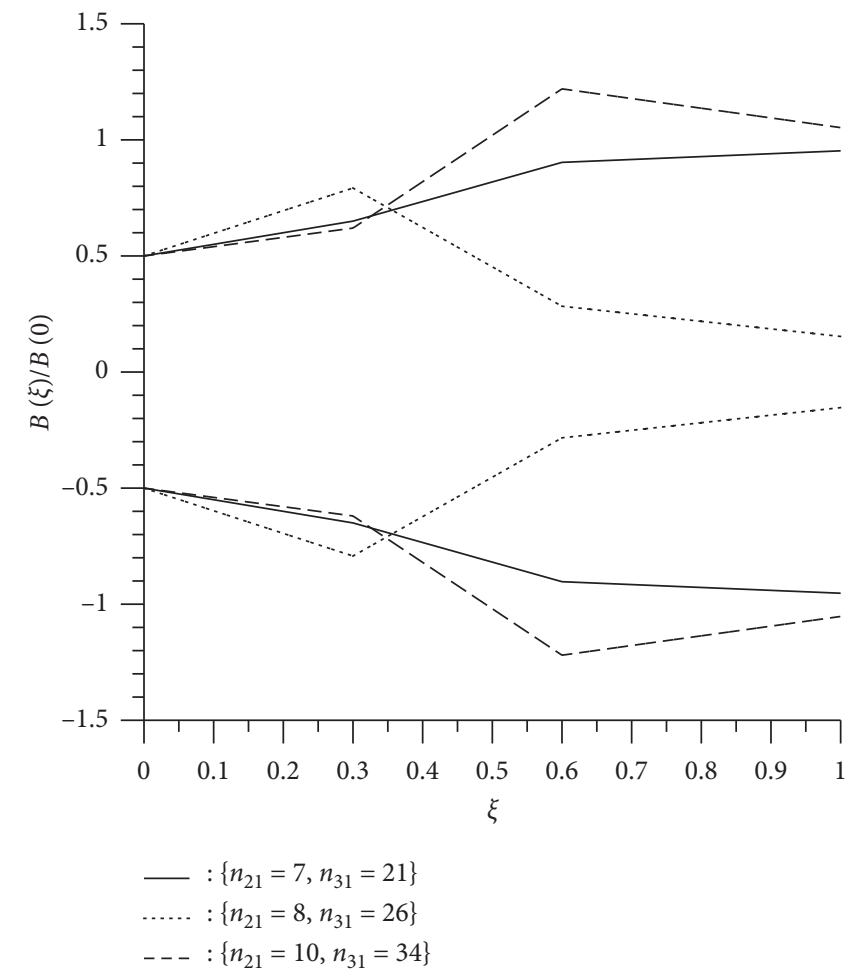

(a)

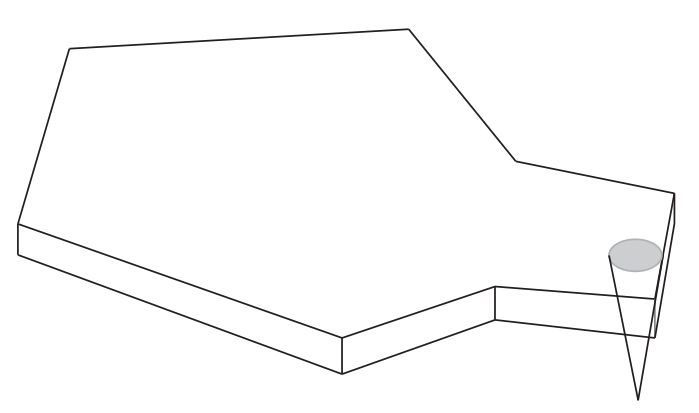

(b)

Figure 3: Stepwise width of a micro probe: (a) width variations of three probes with integer multiples of frequencies; (b) 3D cross section of the probe with frequency ratios $\left\{n_{21}=8, n_{31}=26\right\}$.

$$
m(\xi)=b(\xi)= \begin{cases}\left(1+\lambda_{1} \xi\right), & 0 \leq \xi<\xi_{1} \\ {\left[1+\left(\lambda_{1}-\lambda_{2}\right) \xi_{1}\right]+\lambda_{2} \xi,} & \xi_{1} \leq \xi<\xi_{2} \\ {\left[1+\left(\lambda_{1}-\lambda_{2}\right) \xi_{1}+\left(\lambda_{2}-\lambda_{3}\right) \xi_{2}\right]+\lambda_{3} \xi,} & \xi_{2} \leq \xi<1\end{cases}
$$

Because the coefficients (42) are in polynomial series, the local fundamental solutions can be exactly determined by the Frobenius method [13]. Further, the global semianalytical fundamental solutions can be determined via equation (26).

Without the effect of the interacting force between the tip and sample and considering the probe with the positions of interface $\left\{\xi_{1}=0.3, \xi_{2}=0.6\right\}$, the taper ratios $\left\{\lambda_{1}, \lambda_{2}, \lambda_{3}\right\}$ of AFM cantilevers in the classical theory are tuned such that the second and the third resonance frequencies are integer multiples of the fundamental one for higher harmonic enhancement.

One discovers three types of probe satisfying the integermultiples relation among frequencies which are listed in Table 1. (1) If the taper ratios $\left\{\lambda_{1}, \lambda_{2}, \lambda_{3}\right\}$ are $\{1.0000$,
$1.68730,0.24847\}$, the integer frequency ratios of the probe $\left\{n_{21}, n_{31}\right\}$ are $\{7,21\}$. (2) If the taper ratios $\left\{\lambda_{1}, \lambda_{2}, \lambda_{3}\right\}$ are $\{1.9500,3.39395,-0.65067\}$, the frequency ratios of the probe $\left\{n_{21}, n_{31}\right\}$ are $\{8,26\}$. (3) If the taper ratios $\left\{\lambda_{1}, \lambda_{2}, \lambda_{3}\right\}$ are $\{0.80000,3.99794,-0.83438\}$, the frequency ratios of the probe $\left\{n_{21}, n_{31}\right\}$ are $\{10,34\}$. Figure 3 demonstrates the three profiles of the probe width with different integer multiplication of frequency.

In a similar way, one also discovers three types of probe with the positions of interface $\left\{\xi_{1}=0.4, \xi_{2}=0.7\right\}$, satisfying the integer-multiples relation among frequencies which are listed in Table 2. (1) If the taper ratios $\left\{\lambda_{1}, \lambda_{2}, \lambda_{3}\right\}$ are $\{1.0000,0.68125,-1.72978\}$, the integer frequency ratios of the probe $\left\{n_{21}, n_{31}\right\}$ are $\{6,17\}$. (2) If the taper ratios $\left\{\lambda_{1}, \lambda_{2}, \lambda_{3}\right\}$ are $\{1.0000,1.72422,-1.01995\}$, the frequency ratios $\left\{n_{21}, n_{31}\right\}$ are $\{7,20\}$. (3) If the taper ratios $\left\{\lambda_{1}, \lambda_{2}, \lambda_{3}\right\}$ are $\{1.00000,2.63750,-0.13550\}$, the frequency ratios $\left\{n_{21}, n_{31}\right\}$ are $\{8,23\}$.

When the probe designed without the effect of interacting force between the tip and sample is used to measure the sample topography and material properties, the effect of 
TABLE 2: Three types of probe satisfying the integer-multiples relation among frequencies without the effects of the tip-sample force and the size dependency $\left(\mu=4.0381 \times 10^{-3}, \xi_{1}=0.3\right.$, and $\left.\xi_{2}=0.6\right)$.

\begin{tabular}{cccccccc}
\hline$\lambda_{1}$ & $\lambda_{2}$ & $\lambda_{3}$ & $\omega_{1}$ & $\omega_{2}$ & $\omega_{3}$ & $\left(\omega_{2} / \omega_{1}\right)$ & $\left(\omega_{3} / \omega_{1}\right)$ \\
\hline 1.00000 & 1.68730 & 0.24847 & 2.8896 & 20.2270 & 60.6807 & 7.000 & 21.000 \\
0.19500 & 3.39395 & -0.65067 & 2.4560 & 19.6489 & 63.8571 & 8.000 & 26.000 \\
0.80000 & 3.99794 & -0.83438 & 1.8651 & 18.6517 & 63.4146 & 10.000 & 34.000 \\
\hline
\end{tabular}

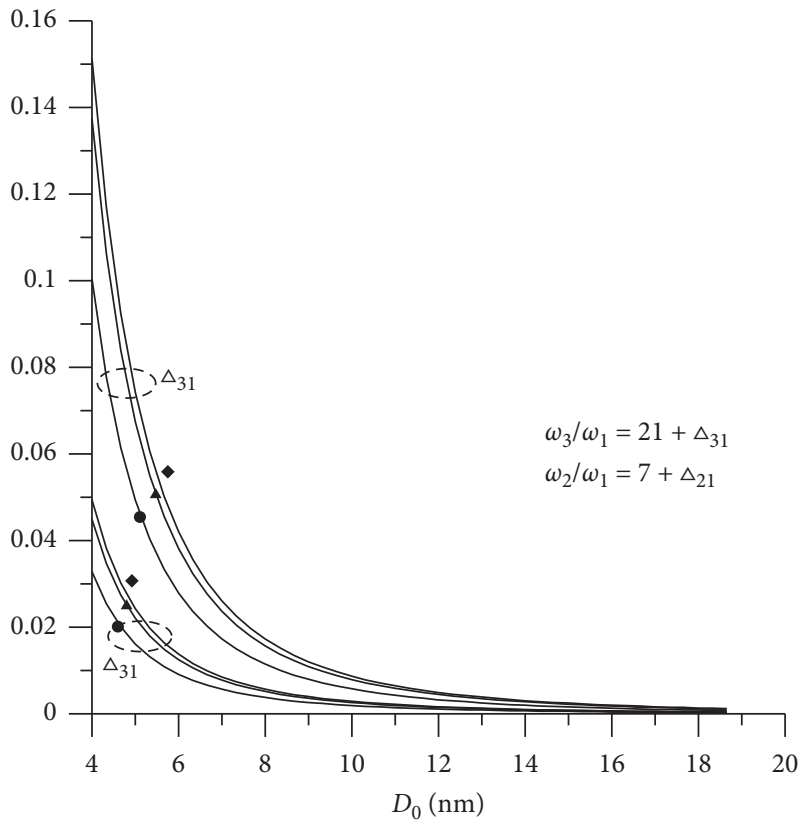

- $\delta=0.0$

$\Delta: \delta=0.1$

- : $\delta=0.5$

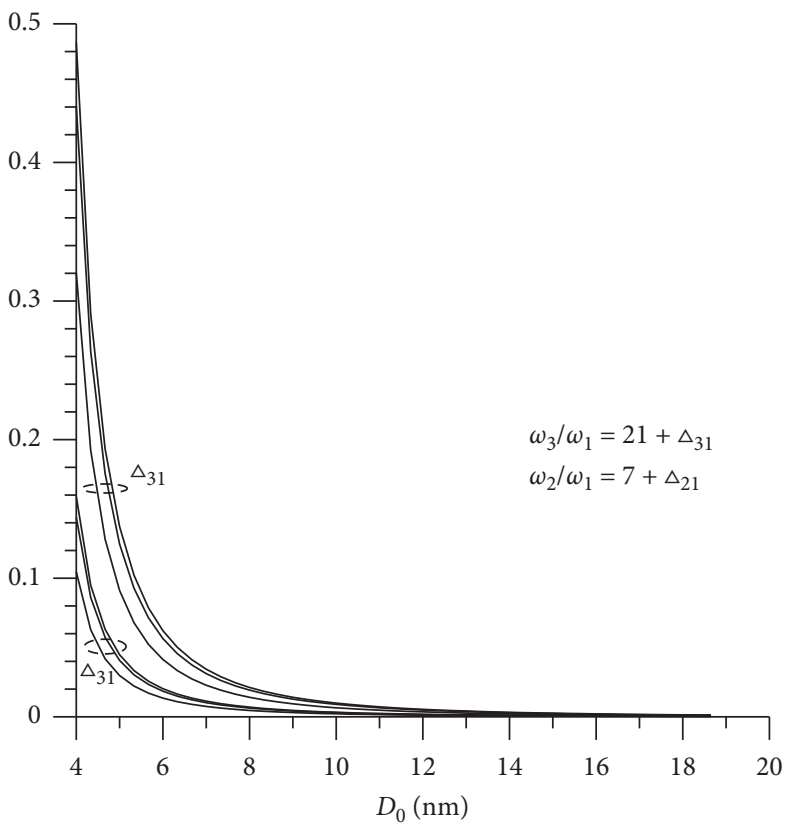

- $\delta=0.0$

$\Delta: \delta=0.1$

- : $\delta=0.5$

(a)

(b)

Figure 4: Effect of the tip-sample distance on the deviation of integer multiples of frequencies: (a) $\bar{w}(L)=1 \mathrm{~nm}$; (b) $\bar{w}(L)=3 \mathrm{~nm}\left(\mathrm{SiO}_{2}\right.$ : $E=70.3 \times 10^{9} \mathrm{~Pa}, \rho=2.5 \times 10^{3} \mathrm{~kg} / \mathrm{m}^{3}, \quad b=45 \mu \mathrm{m}, \quad h=3.5 \mu \mathrm{m}, L=200 \mu \mathrm{m}, \quad m_{\text {tip }}=3.18 \times 10^{-13} \mathrm{~kg}, \mu=4.0381 \times 10^{-3}, \quad \xi_{1}=0.3, \quad \xi_{2}=0.6$, $\lambda_{1}=1.0000, \lambda_{2}=1.68730$, and $\lambda_{3}=0.24847$ ).

interacting force on the deviation of integer multiples of frequencies is investigated.

Figure 4(a) demonstrates that when the tip-sample distance $D_{0}$ is very large, the deviations of integer multiples of frequencies are very small. The smaller the tip-sample distance $D_{0}$ is, the larger the deviations of integer multiples of frequencies are.

The effect of the tip-sample distance $D_{0}$ on the deviation of $\left(\omega_{3} / \omega_{1}\right)$ is significantly more than that of $\left(\omega_{2} / \omega_{1}\right)$.

Moreover, the larger the size-dependency parameter $\delta$ is, the smaller the deviations of integer multiples of frequencies are.

It is observed from Figures 4(a) with tip amplitude $\bar{w}(L)=1 \mathrm{~nm}$ and Figure 3(b) with tip amplitude $\bar{w}(L)=$ $3 \mathrm{~nm}$ that the larger the tip amplitude $\bar{w}(L)$ is, the larger the deviations of integer multiples of frequencies are.

Comparing Figure 4 with $\left\{n_{21}=7, n_{31}=21\right\}$, Figure 5 with $\left\{n_{21}=8, n_{31}=26\right\}$, and Figure 6 with $\left\{n_{21}=10, n_{31}=34\right\}$, it is discovered that the larger the frequency ratios are, the larger the deviations of integer multiples of frequencies are during measuring some samples.

Considering the effect of interacting force at $D_{0}=5 \mathrm{~nm}$ and in the absence of the effect of size dependency, $\delta=0$, the taper ratios $\left\{\lambda_{1}, \lambda_{2}, \lambda_{3}\right\}$ of AFM cantilevers are $\{1.096436,1.68730,0.24847\}$ such that the second and the third resonance frequencies are integer multiples of the fundamental one, $\left\{n_{21}=7, n_{31}=21\right\}$. Figure 7 shows that if the tip-sample distance $D_{0}$ is smaller than $5 \mathrm{~nm}$, the deviations of integer multiples of frequencies are negative. If the tip-sample distance $D_{0}$ is larger than $5 \mathrm{~nm}$, the deviations of integer multiples of frequencies are positive. If the taper ratios $\left\{\lambda_{1}, \lambda_{2}, \lambda_{3}\right\}$ of AFM cantilevers are $\{1.022422,1.68730,0.24847\}$, the deviation at $D_{0}=8 \mathrm{~nm}$ is zero.

Considering the effects of size-dependency, $\delta=0.5$, and the interacting force at $D_{0}=5 \mathrm{~nm}$, Figure 8 shows that if the taper ratios $\left\{\lambda_{1}, \lambda_{2}, \lambda_{3}\right\}$ of AFM cantilevers are $\{0.80000,3.99794,-0.90650\}$, the second and the third resonance frequencies are integer multiples of the fundamental 


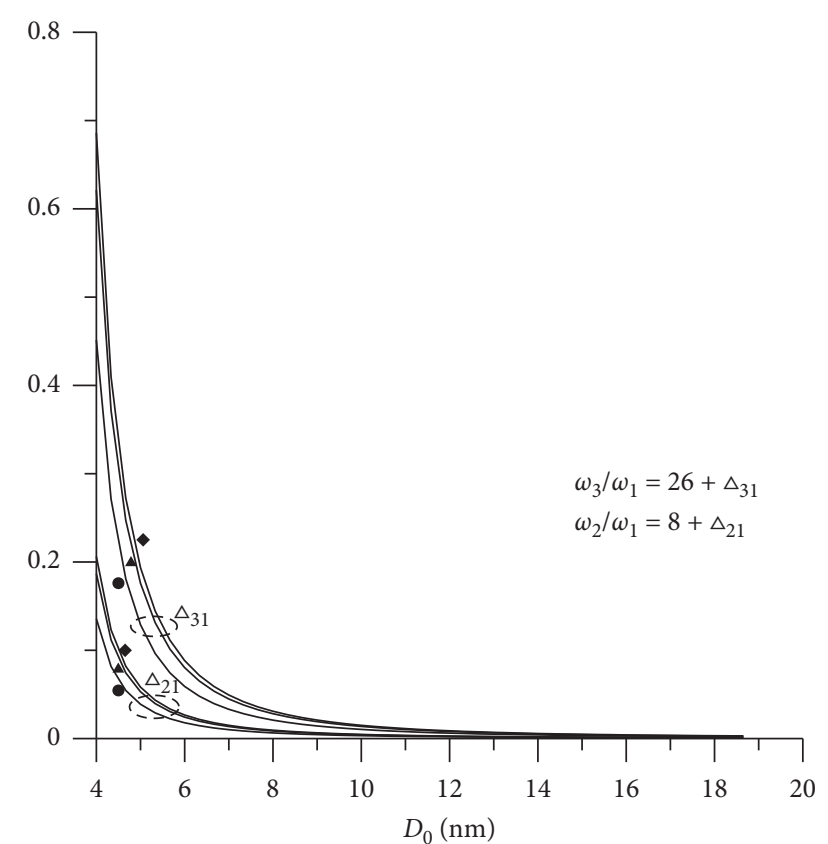

- $: \delta=0.0$

$\Delta: \delta=0.1$

- $: \delta=0.5$

FIGURE 5: Effect of the tip-sample distance on the deviation of integer multiples of frequencies $\left(\mathrm{SiO}_{2}: E=70.3 \times 10^{9} \mathrm{~Pa}\right.$, $\rho=2.5 \times 10^{3} \mathrm{~kg} / \mathrm{m}^{3}, \quad b=45 \mu \mathrm{m}, \quad h=3.5 \mu \mathrm{m}, \quad L=200 \mu \mathrm{m}$, $m_{\text {tip }}=3.18 \times 10^{-13} \mathrm{~kg}, \quad \mu=4.0381 \times 10^{-3}, \quad \xi_{1}=0.3, \quad \xi_{2}=0.6$, $\bar{w}(L)=1 \mathrm{~nm}, \lambda_{1}=0.19500, \lambda_{2}=3.39395$, and $\left.\lambda_{3}=-0.65067\right)$.

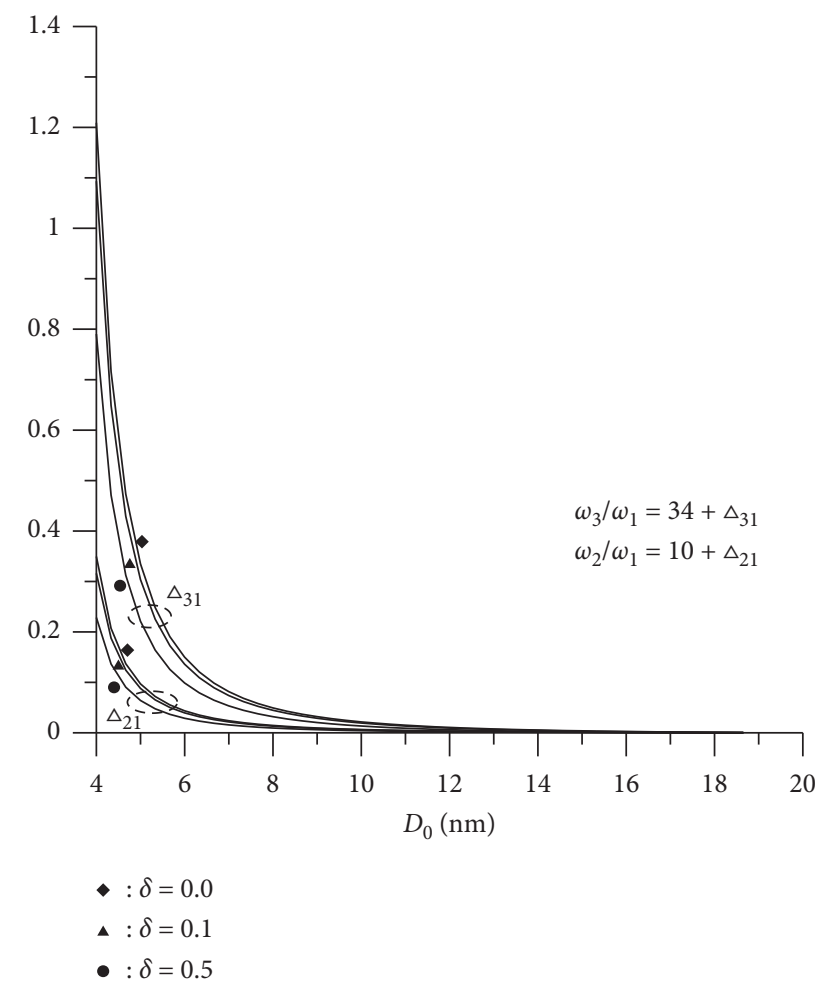

Figure 6: Effect of the tip-sample distance on the deviation of integer multiples of frequencies $\left(\mathrm{SiO}_{2}: E=70.3 \times 10^{9} \mathrm{~Pa}\right.$, $\rho=2.5 \times 10^{3} \mathrm{~kg} / \mathrm{m}^{3}, \quad b=45 \mu \mathrm{m}, \quad h=3.5 \mu \mathrm{m}, \quad L=200 \mu \mathrm{m}$, $m_{\text {tip }}=3.18 \times 10^{-13} \mathrm{~kg}, \quad \mu=4.0381 \times 10^{-3}, \quad \xi_{1}=0.3, \quad \xi_{2}=0.6$, $\bar{w}(L)=1 \mathrm{~nm}, \lambda_{1}=0.80000, \lambda_{2}=3.99794$, and $\left.\lambda_{3}=-0.83438\right)$.

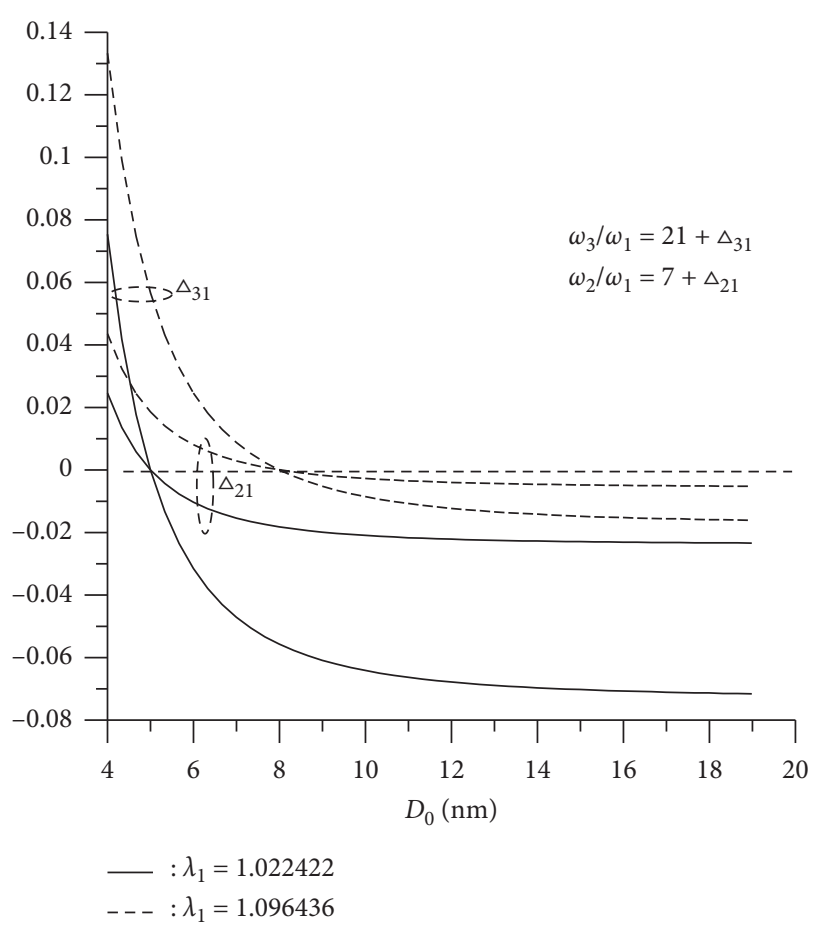

FIgURE 7: Effect of the tip-sample distance on the deviation of integer multiples of frequencies where $\left(\omega_{2} / \omega_{1}\right)=7$ and $\left(\omega_{3} / \omega_{1}\right)=$ 21 at $D_{0}=5$ or $8 \mathrm{~nm}\left(\mathrm{SiO}_{2}: E=70.3 \times 10^{9} \mathrm{~Pa}, \rho=2.5 \times 10^{3} \mathrm{~kg} / \mathrm{m}^{3}\right.$, $b=45 \mu \mathrm{m}, \quad h=3.5 \mu \mathrm{m}, \quad L=200 \mu \mathrm{m}, \quad m_{\text {tip }}=3.18 \times 10^{-13} \mathrm{~kg}$, $\mu=4.0381 \times 10^{-3}, \quad \xi_{1}=0.3, \quad \xi_{2}=0.6, \lambda_{2}=1.68730, \lambda_{3}=0.24847$, $\left(\omega_{3} / \omega_{1}\right)=21+\Delta_{31}, \delta=0$, and $\left.\bar{w}(L)=1 \mathrm{~nm}\right)$.

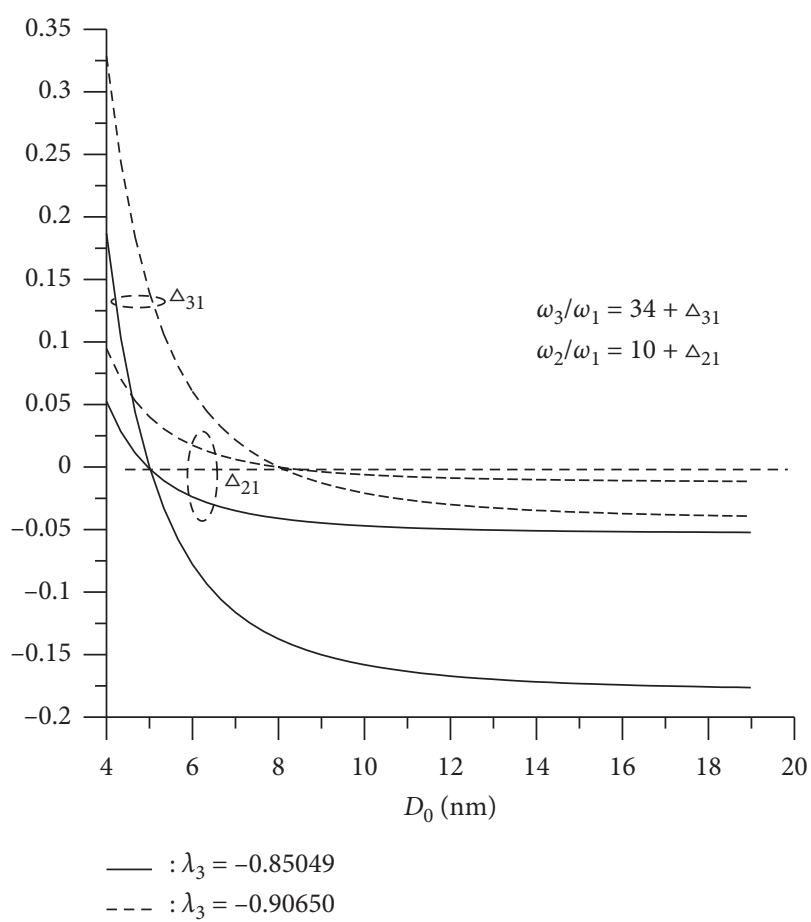

FIgURE 8: Effect of the tip-sample distance on the deviation of integer multiples of frequencies where $\left(\omega_{2} / \omega_{1}\right)=10$ and $\left(\omega_{3} / \omega_{1}\right)=$ 34 at $D_{0}=5$ or $8 \mathrm{~nm}\left(\mathrm{SiO}_{2}: E=70.3 \times 10^{9} \mathrm{~Pa}, \rho=2.5 \times 10^{3} \mathrm{~kg} / \mathrm{m}^{3}\right.$, $b=45 \mu \mathrm{m}, \quad h=3.5 \mu \mathrm{m}, \quad L=200 \mu \mathrm{m}, \quad m_{\text {tip }}=3.18 \times 10^{-13} \mathrm{~kg}$, $\mu=4.0381 \times 10^{-3}, \quad \xi_{1}=0.3, \quad \xi_{2}=0.6, \quad \lambda_{1}=0.80000, \lambda_{2}=3.99794$, $\delta=0.5$, and $\bar{w}(L)=1 \mathrm{~nm})$. 
TABLE 3: Comparison of the dimensionless frequencies determined by the proposed probe and similarity formula (46) at the tip-sample distance $D_{0}=5 \mathrm{~nm}\left(\mu=4.0381 \times 10^{-3}, \xi_{1}=0.3, \xi_{2}=0.6, \lambda_{1}=1.096436, \lambda_{2}=1.68730, \lambda_{3}=0.24847,\left(\omega_{2} / \omega_{1}\right)=7+\Delta_{21},\left(\omega_{3} / \omega_{1}\right)=21+\Delta_{31}\right.$, $c_{v, \text { stress }}=(1+\delta) c_{v, \text { classical }}, D_{0}=5 \mathrm{~nm}$, and $\left.\bar{w}(L)=1 \mathrm{~nm}\right)$.

\begin{tabular}{cccccc}
\hline$\delta$ & $\omega_{1, \text { classical }}$ & Proposed method & $\omega_{1, \text { stress }}$ & $\Delta_{21}=\left(\omega_{2} / \omega_{1}\right)-7$ & $\Delta_{31}=\left(\omega_{3} / \omega_{1}\right)-21$ \\
\hline 0.0 & 2.8827 & 2.8827 & Equation $(42)$ & 0.000 & 0.000 \\
0.1 & - & 3.0234 & 2.8827 & 0.000 & 0.000 \\
0.2 & - & 3.1578 & 3.0234 & 0.000 & 0.000 \\
0.3 & - & 3.2867 & 3.1578 & 0.000 & 0.000 \\
0.4 & - & 3.4108 & 3.2867 & 0.000 & 0.000 \\
0.5 & - & 3.5305 & 3.4108 & 0.000 & 0.000 \\
0.6 & - & 3.6463 & 3.5305 & 0.000 & 0.000 \\
0.7 & - & 3.7585 & 3.6463 & 0.000 & 0.000 \\
0.8 & - & 3.8675 & 3.7585 & 0.000 & 0.000 \\
0.9 & - & 4.0735 & 3.9735 & 0.000 & 0.000 \\
1.0 & & & 4.0767 & & \\
\hline
\end{tabular}

TABLE 4: Comparison of the dimensionless frequencies determined by the proposed probe and similarity formula (46) at the tip-sample distance $D_{0}=8 \mathrm{~nm}\left(\mu=4.0381 \times 10^{-3}, \xi_{1}=0.3, \xi_{2}=0.6, \lambda_{1}=1.022422, \lambda_{2}=1.68730, \lambda_{3}=0.24847,\left(\omega_{2} / \omega_{1}\right)=7+\Delta_{21},\left(\omega_{3} / \omega_{1}\right)=21+\Delta_{31}\right.$, $c_{v, \text { stress }}=(1+\delta) c_{v, \text { classical }}, D_{0}=8 \mathrm{~nm}$, and $\left.\bar{w}(L)=1 \mathrm{~nm}\right)$.

\begin{tabular}{cccccc}
\hline$\delta$ & $\omega_{1, \text { classical }}$ & Proposed method & $\omega_{1, \text { stress }}$ & $\Delta_{21}=\left(\omega_{2} / \omega_{1}\right)-7$ & $\Delta_{31}=\left(\omega_{3} / \omega_{1}\right)-21$ \\
\hline 0.0 & 2.8879 & 2.8879 & Equation $(42)$ & 0.000 & 0.000 \\
0.1 & - & 3.0289 & 2.8879 & 0.000 & 0.000 \\
0.2 & - & 3.1636 & 3.0289 & 0.000 & 0.000 \\
0.3 & - & 3.2928 & 3.1636 & 0.000 & 0.000 \\
0.4 & - & 3.4171 & 3.2927 & 0.000 & 0.000 \\
0.5 & - & 3.5370 & 3.4171 & 0.000 & 0.000 \\
0.6 & - & 3.6530 & 3.5370 & 0.000 & 0.000 \\
0.7 & - & 3.7654 & 3.6530 & 0.000 & 0.000 \\
0.8 & - & 3.8746 & 3.7654 & 0.000 & 0.000 \\
0.9 & - & 3.9808 & 3.8746 & 0.000 & 0.000 \\
1.0 & - & 4.0842 & 3.9808 & 0.000 &
\end{tabular}

one, $\left\{n_{21}=10, n_{31}=34\right\}$. If the tip-sample distance $D_{0}$ is smaller than $5 \mathrm{~nm}$, the deviation of integer multiples of frequencies is negative. If the tip-sample distance $D_{0}$ is larger than $5 \mathrm{~nm}$, the deviation of integer multiples of frequencies is positive. If the taper ratios $\left\{\lambda_{1}, \lambda_{2}, \lambda_{3}\right\}$ of AFM cantilevers are $\{0.80000,3.99794,-0.85049\}$, the deviation at $D_{0}=8 \mathrm{~nm}$ is zero.

Considering the tip-sample distance $D_{0}=5 \mathrm{~nm}$, the taper ratios $\left\{\lambda_{1}, \lambda_{2}, \lambda_{3}\right\}$ of AFM cantilevers in the classical theory are tuned such that the second and the third resonance frequencies are integer multiples of the fundamental one, $\left\{n_{21}=7, n_{31}=21\right\}$. With the effect of size dependency, Table 3 reveals that the proposed probe has the same integermultiples relation of frequency at the tip-sample distance $D_{0}=5 \mathrm{~nm}$. The numerical results determined by the proposed method and similarity formula (44) are consistent. The fact is also demonstrated in Table 4 with the tip-sample distance $D_{0}=8 \mathrm{~nm}$.

\section{Conclusion}

With the effect of the interacting tip-sample force, the integermultiples relations among frequencies of nonuniform rectangular AFM probes in the classical theory and the modified couple stress one are discovered. When the thickness of rectangular cross section of the beam is constant, the integermultiples relations in different theories are the same in spite of the size-dependency parameter $\delta$. The stepwise width of probe can be tuned to match the requirement of integer multiples of frequencies. Based on the conventional harmonic probe designed without the effect of the tip-sample force, the effect of interacting force on the deviation of integer multiples of frequencies is investigated as follows:

(1) The smaller the tip-sample distance $D_{0}$ is, the larger the deviations of integer multiples of frequencies are.

(2) The effect of the tip-sample distance $D_{0}$ on the deviation of $\left(\omega_{3} / \omega_{1}\right)$ is significantly more than that of $\left(\omega_{2} / \omega_{1}\right)$.

(3) The larger the size-dependency parameter $\delta$ is, the smaller the deviations of integer multiples of frequencies are.

(4) The larger the tip amplitude $\bar{w}(L)$ is, the larger the deviations of integer multiples of frequencies are.

Finally, one can tune the taper ratios of the AFM cantilevers such that the integer multiplies relations for some preferred tip-sample distance $D_{0}$. Moreover, under 
similarity relations (38)-(42), the probes with different sizedependency parameter $\delta$ have the same integer-multiples relation of frequency at the same tip-sample distance $D_{0}$.

\section{Nomenclature}

A: Cross-sectional area of the beam

$b$ : Dimensionless bending rigidity, $\{I(x) / I(0)$

$c_{v}$ : Dimensionless parameter of van der Waals force

E: $\quad$ Young's modulus

I: $\quad$ Area inertia of the beam

$L: \quad$ Lengths of the beam

$\ell_{c}$ : $\quad$ Material length scale parameter

$m$ : Dimensionless mass of the beam, $\{A(x) / A(0)$

$m_{\text {tip }}:$ Tip mass of the beam

$n_{21}$, The integer frequency ratios, $\left(\omega_{2} / \omega_{1}\right),\left(\omega_{3} / \omega_{1}\right)$

$n_{31}:$

$x, y, z$ : Coordinate of the beam

$t$ : Time variable

$W$ : Transverse displacements of the beam and membrane

$w$ : Dimensionless transverse displacements of the beam and plate, $(W / L)$

v: $\quad$ Poisson's ratio

$\mu: \quad$ Shear modulus

$\mu_{\text {tip }}: \quad$ Dimensionless tip mass of the beam, $\left\{m_{\text {tip }} / \rho A(0) L\right.$

$\xi: \quad$ Dimensionless principal beam coordinate, $(x / L)$

$\Omega$ : $\quad$ Natural frequency

$\omega$ : Dimensionless natural frequency, $\Omega L^{2} \sqrt{\{\rho A(0) / E I(0)}$

$\rho: \quad$ Mass density

$\sigma_{i j}: \quad$ Stress tensor

$\varepsilon_{i j}: \quad$ Strain tensor

$m_{i j}: \quad$ Couple stress tensor

$\chi_{i j}$ : Antisymmetric curvature tensor

$\tau: \quad$ Dimensionless time, $\left(t / L^{2}\right) \sqrt{\{E I(0) / \rho A(0)}$.

\section{Data Availability}

The data used to support the findings of this study are included within the article.

\section{Conflicts of Interest}

The authors declare that they have no conflicts of interest.

\section{Acknowledgments}

The support of the Ministry of Science and Technology of Taiwan, R. O. C., is gratefully acknowledged (grant nos. MOST 108-2221-E-168-009 and MOST 109-2221-E-168008).

\section{References}

[1] S.-M. Lin, C.-T. Liauh, W.-R. Wang, and S.-H. Ho, "Analytical solutions of the first three frequency shifts of AFM nonuniform probe subjected to the Lennard-Jones force," Ultramicroscopy, vol. 106, no. 6, pp. 508-515, 2006.
[2] S.-M. Lin, "Analytical solutions of the frequency shifts of several modes in dynamic force microscopy subjected to AC electrostatic force," IEEE Transactions On Nanotechnology, vol. 6, no. 4, pp. 404-412, 2007.

[3] S. M. Lin, C. T. Liauh, W. R. Wang, and S. H. Ho, "Analytical solutions of the frequency shifts of several modes in nonuniform probes scanning an inclined surface, subjected to the Lennard-Jones force," International Journal of Solids and Structures, vol. 44, no. 3-4, pp. 799-810, 2007.

[4] S.-M. Lin and W.-R. Wang, "Frequency shifts and analysis of AFM accompanying with coupled flexural-torsional motions," International Journal of Solids and Structures, vol. 46, no. 24, pp. 4231-4241, 2009.

[5] Z. Li, T. Shi, and X. Qi, "An optimized harmonic probe with tailored resonant mode for multifrequency atomic force microscopy," Advances in Mechanical Engineering, vol. 10, no. 11, pp. 1-7, 2018.

[6] Y. Hou, C. Ma, W. Wang, and Y. Chen, "Binary coded cantilevers for enhancing multi-harmonic atomic force microscopy," Sensors and Actuators A: Physical, vol. 300, Article ID 111668, 2019.

[7] F. Gramazio, M. Lorenzoni, F. Pérez-Murano, L. Evangelio, and J. Fraxedas, "Quantification of nanomechanical properties of surfaces by higher harmonic monitoring in amplitude modulated AFM imaging," Ultramicroscopy, vol. 187, pp. 20-25, 2018.

[8] H. Li, Y. Chen, and L. Dai, "Concentrated-mass cantilever enhances multiple harmonics in tapping-mode atomic force microscopy," Applied Physics Letters, vol. 92, no. 15, p. 151903, 2008.

[9] W. Zhang, Y. Chen, and J. Chu, "Cantilever optimization for applications in enhanced harmonic atomic force microscopy," Sensors and Actuators A: Physical, vol. 255, pp. 54-60, 2017.

[10] B. Zhu, S. Zimmermann, X. Zhang, and S. Fatikow, "A systematic method for developing harmonic cantilevers for atomic force microscopy," ASME Journal of Mechanical Design, vol. 139, Article ID 012303, 2017.

[11] J. Cai, Q. Xia, Y. Luo, L. Zhang, and M. Y. Wang, “A variablewidth harmonic probe for multifrequency atomic force microscopy," Applied Physics Letters, vol. 106, Article ID 071901, 2015.

[12] R. Sriramshankar and G. R. Jayanth, "Design and evaluation of torsional probes for multifrequency atomic force microscopy," IEEE/ASME Transactions on Mechatronics, vol. 20, no. 4, pp. 1843-1853, 2015.

[13] S. M. Lin and C. Y. Chang, "Size-dependency effect on design of harmonic AFM probe in the modified couple stress theory," Mechanics of Advanced Materials and Structures, pp. 1-9, 2020.

[14] A. F. Payam, "Dynamic modeling and sensitivity analysis of dAFM in the transient and steady state motions," Ultramicroscopy, vol. 169, pp. 55-61, 2016.

[15] S. Santos and K. R. Gaderlab, "Single-cycle atomic force microscope force reconstruction: resolving time-dependent interactions," New Journal of Physics, vol. 15, Article ID 083034, 2013.

[16] D. R. Sahoo, A. Sebastian, and M. V. Salapaka, "Harnessing the transient signals in atomic force microscopy," International Journal of Robust and Nonlinear Control, vol. 15, no. 16, pp. 805-820, 2005.

[17] P. I. Chang, P. Huang, J. Maeng, and S. B. Andersson, "Local raster scanning for high speed imaging of biopolymers in atomic force microscopy," Review of Scientific Instruments, vol. 82, no. 6, Article ID 063703, 2011. 
[18] S.-M. Lin, C.-L. Tsai, and H.-C. Lee, "Effects of size dependency and axial deformation on the dynamic behavior of an AFM subjected to van der Waals force based on the modified couple stress theory," Precision Engineering, vol. 56, pp. 203-210, 2019.

[19] S. M. Lin and C.-Y. Chang, "Resonance-size parameter relationship and dynamics of an AFM subjected to multi-mode excitation and based on the modified couple stress theory," Mathematical Problem in Engineering, vol. 2019, Article ID 3147863, 10 pages, 2019.

[20] S. M. Lin, C. T. Liauh, and W. R. Wang, "Assessment of different theories about size dependency effect on the resonant frequency of an AFM subjected to van der Waals force," Mechanics of Advanced Materials and Structures, pp. 1-12, 2019.

[21] T. Murmu and S. Adhikari, "Nonlocal elasticity based vibration of initially pre-stressed coupled nanobeam systems," European Journal of Mechanics A/Solids, vol. 34, pp. 52-62, 2012.

[22] R. Ansari, K. Hosseini, A. Darvizeh, and B. Daneshian, "A sixth-order compact finite difference method for non-classical vibration analysis of nanobeams including surface stress effects," Applied Mathematics and Computation, vol. 219, no. 10, pp. 4977-4991, 2013.

[23] S. M. Lin, W. R. Wang, and S. Y. Lee, "The dynamic analysis of non-uniformly pretwisted timoshenko beams with elastic boundary conditions," International Journal of Mechanical Sciences, vol. 43, no. 10, pp. 2385-2405, 2001. 